\title{
Synthesis and Characterization of Poly(acrylic acid) Brushes: "Grafting-Onto" Route
}

\section{Guillaume Sudre, ${ }^{\mathrm{a}}$ Elodie Siband, ${ }^{\mathrm{a}}$ Dominique Hourdet, ${ }^{\mathrm{a}}$ Costantino Creton, ${ }^{a}$ Fabrice Cousin, ${ }^{b}$ Yvette $\operatorname{Tran}^{\mathrm{a}}{ }^{*}$}

a Soft Matter Sciences and Engineering, UMR 7615, ESPCI-CNRS-UPMC, 10 rue Vauquelin, 75231 Paris Cedex 05, France

b Laboratoire Léon Brillouin, CEA-CNRS, Saclay 91191 Gif-sur-Yvette Cedex, France

* Corresponding author: yvette.tran@espci.fr

Published in Macromolecular Chemistry and Physics:

Synthesis and Characterization of Poly(acrylic acid) Brushes: "Grafting onto" Route, Sudre G., Siband E., Hourdet D., Creton C., Cousin F., Tran Y., Macromolecular Chemistry \& Physics, 2012, 213(3), 293300.

Link to the publisher version: http://dx.doi.org/10.1002/macp.201100394

Creative Commons Attribution Non-Commercial No Derivatives License 


\begin{abstract}
A guideline for the synthesis of poly(acrylic acid) brushes on planar silica surfaces by the "graftingonto" approach is described. It is demonstrated that some thermal precautions must be taken to obtain extended brushes. It is also shown that neutron reflectivity is well suited for the characterization of each step of the synthesis, while it is (unfortunately) rarely used for that purpose. The steps are the following: first, the substrates are covered with a self-assembled monolayer of epoxy-terminated molecules; then, the poly(tert-butyl acrylate) brushes are built using preformed and end-functionalized chains; finally, the deprotection of the ester group is performed using a pyrolysis reaction to convert the poly(tert-butyl acrylate) brushes into poly(acrylic acid) brushes.
\end{abstract}

\title{
Graphical abstract
}

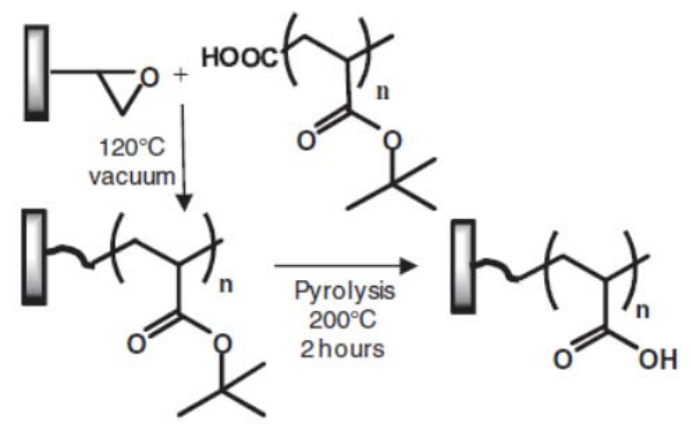




\section{Introduction}

Polymer brushes, in which polymer chains are densely attached by one end to a surface or interface, are extensively used for the improvement of adhesion, wetting properties or colloidal stabilization. Some synthetic strategies have been developed to functionalize silicon surfaces with polymer brushes in order to design smart surfaces with switchable and adaptative properties and to generate patterned surfaces in the areas of nanotechnology.[1-6] Among different types of possible polymers, poly(acrylic acid) brushes are interesting in their own right, with biological applications in biosensors, cell adhesion and protein adsorption.[7-9] As a result, their specific synthesis has attracted increasing attention during the last decade.

Covalently surface-attached polymer chains can be synthesized by the "grafting-onto" strategy, where preformed chains containing appropriate end-functionalized groups are reacted with the surface to obtain the desired brush. Chains with chlorosilane termination have been directly anchored to silicon-wafer substrates by the reaction of chlorosilane with the hydroxyl surface functionality.[10, 11] A more versatile two-step process was developed by Minko et al. [5, 6, 12-14] in which $\gamma$-glycidoxypropyltrimethoxysilane was first grafted onto silicon wafers and carboxylterminated chains were then anchored on the modified silica surface. This method was also used successfully for the synthesis of mixed brushes.

Polyacid brushes can also be synthesized by the "grafting from" approach using controlled radical polymerization techniques such as atom-transfer radical polymerization (ATRP). However, ATRP of acrylic acid is problematic since the carboxylic acid may react with metal complexes to form catalytically ineffective metal carboxylates. It follows that the synthesis of polyacid brushes frequently includes the polymerization of an ester-containing monomer and the subsequent deprotection of the ester group by a hydrolysis [15-17] or pyrolysis [18-20] reaction.

In our group, we are interested in the synthesis of polymer brushes, as well as their careful characterization using several complementary techniques that allow the determination of the chemical and structural properties of the brushes. Some of us recently reported the synthesis of weak polybase brushes [21] and polyampholyte brushes by surface-initiated ATRP and their characterization.[22, 23] In this paper, we report the synthesis of poly(acrylic acid) brushes obtained by the "grafting-onto" approach. To the best of our knowledge, the different steps needed for the synthesis of poly(carboxylic acid) brushes using the "grafting-onto" method have never been carefully characterized, even if this straightforward approach does not require very sophisticated chemistry. It is also rather cheap since it requires only small amounts of (commercial) polymers. Here, we demonstrate that neutron reflectivity can be used to characterize each step of the synthesis, in addition to the usual techniques, such as ellipsometry and infrared spectrometry. The purpose of this paper is to show that some thermal precautions must be taken to obtain extended poly(acrylic acid) brushes. Thus, a guideline for the synthesis of poly(acrylic acid) brushes by the "grafting-onto" approach can be drawn. 


\section{Experimental Section}

\subsection{Chemical Products and Silicon Substrates}

Three samples of carboxyl-terminated poly(tert-butyl acrylate) (PtBuA-COOH) with high and low molecular weights $\left(M_{\mathrm{n}}=42000 \mathrm{~g} \mathrm{~mol}^{-1}, \ominus=1.12 ; M_{\mathrm{n}}=6500 \mathrm{~g} \mathrm{~mol}^{-1}, \ominus=1.08\right.$; and $M_{\mathrm{n}}=4200 \mathrm{~g} \mathrm{~mol}^{-1}$ , $\ominus=1.25$ ) were purchased from Polymer Source. 3-Glycidoxypropyltrimethoxysilane (GPS) (Gelest, 97\%), anhydrous toluene (Aldrich, 99.8\%) and tetrahydrofuran (THF) (SDS, 95\%) were used as received.

All of the silicon wafers were purchased from ACM. $100 \mathrm{~mm} \times 50 \mathrm{~mm} \times 10 \mathrm{~mm}$ monocrystals were used for the neutron reflectivity measurements. $70 \mathrm{~mm} \times 10 \mathrm{~mm} \times 1.5 \mathrm{~mm}$ trapezoidal crystals (with an angle of $45^{\circ}$ ) were used for Fourier transform-IR attenuated-total-reflection (FTIR-ATR) spectroscopy measurements. The silicon substrates were cleaned by treatment with freshly prepared "piranha" solution (70:30 v/v concentrated $\mathrm{H}_{2} \mathrm{SO}_{4} / 30 \%$ aqueous $\mathrm{H}_{2} \mathrm{O}_{2}$ ) at $150{ }^{\circ} \mathrm{C}$ for 30 min (caution: piranha solution is extremely corrosive). The substrates were then rinsed with pure water (Millipore, resistivity $\geq 18.2 \mathrm{M} \Omega \mathrm{cm}$ ), cleaned by ultrasound in water for $1 \mathrm{~min}$ and dried under nitrogen.

\subsection{Synthesis}

The poly(tert-butyl acrylate) (PtBuA) brushes were synthesized by the "grafting-onto" method using a two-step procedure, as illustrated in Scheme 1. The first step consisted of the formation of a silane self-assembled monolayer by exposing freshly cleaned silicon wafers to a 2 vol\% GPS solution in anhydrous toluene for $5 \mathrm{~h}$. In the second step, the grafting of the PtBuA chains was obtained after the esterification of the PtBuA-COOH end-groups with the surface-attached GPS.[13] The polymer film was spread on the GPS monolayer by spin-coating from a $1 \mathrm{wt} \% \mathrm{PtBuA}-\mathrm{COOH}$ solution in THF and heated at $120^{\circ} \mathrm{C}$ in a vacuum oven for $24 \mathrm{~h}$. The silicon wafers were then rinsed extensively with THF to remove any ungrafted polymer chains. The substrates were finally sonicated in THF for 2 min and dried under a nitrogen stream. This grafting process was performed first for the "long" chains $\left(M_{\mathrm{n}}=42000 \mathrm{~g} \mathrm{~mol}^{-1}\right)$ and then for the "very short" chains $\left(M_{\mathrm{n}}=4200 \mathrm{~g} \mathrm{~mol}^{-1}\right)$. This added carpet of very short chains was used to cover the GPS silane molecules that were not linked to the long PtBuA chains.

The PtBuA brushes were converted into PAA brushes by a pyrolysis reaction. The silicon wafers functionalized by the PtBuA brushes were placed in an oven preheated to $200{ }^{\circ} \mathrm{C}$. They were then allowed to heat under vacuum for $2 \mathrm{~h}$. 


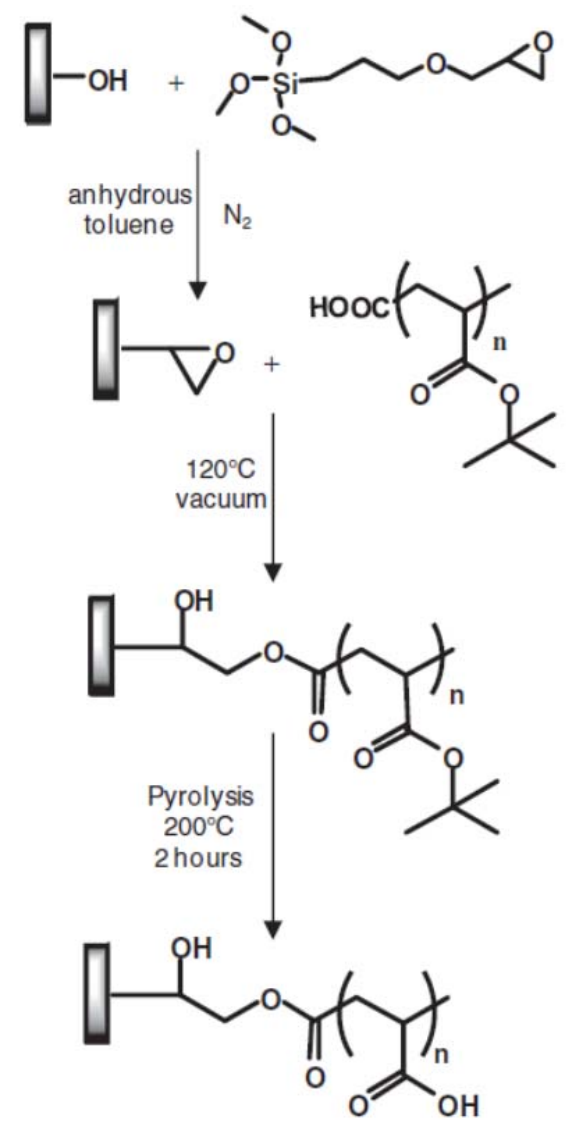

Scheme 1. Synthesis of the poly(acrylic acid) brushes using the "grafting-onto" route. First, the epoxyterminated silane (3-glycidoxypropyltrimethoxysilane) was covalently attached to the silicon substrate in a selfassembled monolayer. The second step is the grafting of carboxyl-functionalized poly(tert-butyl acrylate) by a spin-coating/thermal annealing methodology. The final step is the conversion of the poly(tert-butyl acrylate) brush into a poly(acrylic acid) brush by a pyrolysis reaction.

\subsection{Characterization}

Ellipsometry measurements were performed using a Sentech SE 400 apparatus. The light source was a He-Ne laser $(\lambda=632.8 \mathrm{~nm})$ and the angle of incidence was set to $70^{\circ}$. A multilayer model for a flat film was used for the calculation of the thicknesses of the silica, initiator and grafted polymer layers from the experimentally measured ellipsometric angles $\psi$ and $\Delta$. The refractive indices used for the calculations were 3.874 for the silicon substrate, 1.460 for the native silica layer, 1.460 for the GPS, 1.466 for the PtBuA and 1.527 for the PAA brushes.

The polymer brushes were characterized by ellipsometry to measure their dry thickness, $v(\AA)$. The grafting density $\sigma$, which corresponds to the number of chains per unit area, was calculated from the dry thickness and the molecular weight $M_{\mathrm{n}}$ using Equation 1:

$$
\sigma=\frac{\gamma \rho N_{\mathrm{A}}}{M_{\mathrm{n}}}
$$

In Equation 1, $\rho$ is the density and $M_{\mathrm{n}}$ is the number-average molecular weight of the polymer. The density values used for the calculations were $\rho=1.050 \mathrm{~g} \mathrm{~cm}^{-3}$ for PtBuA and $\rho=1.080 \mathrm{~g} \mathrm{~cm}^{-3}$ for PAA. 
Neutron reflectivity measurements were performed at the silicon-liquid interface using the EROS reflectometer at the Laboratoire Léon Brillouin, CEA-Saclay (France). The experimental procedure and setup are described in detail in previous papers.[10,21] Neutron reflectivity measurements were performed with protonated polymer brushes and deuterated water in order to determine the monomer density profile of the brushes. The neutron reflectivity is sensitive to the scattering length density profile perpendicular to the interface, $N b(z)$. A reliable, model-independent method was chosen to determine $\mathrm{Nb}(z)$. The brush was modeled as a set of layers, each characterized by a fixed thickness and scattering length density. Two adjacent layers were connected using error functions of fixed width to get a continuous profile. The procedure consisted of choosing a scattering length density profile and finding the corresponding parameters for which the calculated reflectivity curve fitted the experimental reflectivity data the best. This reliable method allowed the determination of a continuous scattering length density profile, without making any assumptions about its analytical form. The monomer volume fraction profile $\varphi(z)$ was then deduced from $N b(z)$. Calculated from the

volume fraction profile, the length $\gamma=\int_{0}^{\infty} \varphi(z) d z$ is an important parameter because it is independent of the shape of $\varphi(z)$. It corresponds to the thickness of the dry layer and has to be compared with values measured by another technique, such as ellipsometry.

The FTIR-ATR spectra were recorded using a Magna IR 550 (Nicolet) apparatus with a mercurycadmium-telluride (MCT) detector cooled with liquid nitrogen. The spectra were recorded with a resolution of $2 \mathrm{~cm}^{-1}$ and a 256 -scan accumulation.

Thermogravimetric analyses (TGA) were performed using an SDT Q600 instrument from TA Instruments. The samples were first equilibrated at $30{ }^{\circ} \mathrm{C}$ and then heated at $5{ }^{\circ} \mathrm{C} \mathrm{min}^{-1}$ until the temperature reached $200^{\circ} \mathrm{C}$. The sample purge flow was set to $100 \mathrm{~mL}$ of nitrogen per minute.

\section{Results and Discussion}

\subsection{The Synthesis of the Poly(tert-butyl acrylate) Brushes}

The poly(tert-butyl acrylate) brushes were synthesized by following the "grafting-onto" strategy, which consisted of the attachment of preformed and end-functionalized PtBuA chains on the surface (Scheme 1). The "grafting-onto" method is convenient since it requires commercial chemicals such as the GPS silane for the formation of the self-assembled monolayer in the first step and the endfunctionalized PtBuA-COOH in the second step. In addition, only small amounts of PtBuA-COOH were used since the polymer films were obtained by spin-coating. The $500 \mathrm{~nm}$ thick films formed by spreading a $1 \mathrm{wt} \%$ polymer solution was a sufficient reservoir for the grafting of chains on the surface. The thickest dry brush obtained after removing the ungrafted chains was about $93 \AA$ for the PtBuA chains with a molecular weight of $42000 \mathrm{~g} \mathrm{~mol}^{-1}$, resulting in $\sigma=0.14 \mathrm{~nm}^{-2}$. The highest grafting density obtained here by the "grafting-onto" method is lower than that reached by the "grafting-from" approach: because of steric effects, the diffusion of the reactive species for the "grafting-from" approach (the monomer) close to the surface is much higher than that of the whole chain, which is needed in the "grafting-onto" approach. However, in using the "grafting-onto" method, we could also obtain PtBuA brushes of different grafting densities by varying the reaction time or the proportion of end-functionalized chains, as indicated in Table 1. The difference between the brush thickness (or grafting density) for 1 and $24 \mathrm{~h}$ of reaction was modest ( 83 and $93 \AA$ ), while a 
PtBuA brush with a twice-lower density $\left(0.060 \mathrm{~nm}^{-2}\right.$ versus $\left.0.125 \mathrm{~nm}^{-2}\right)$ was obtained with $10 \%$ of reactive chains instead of $100 \%$.

Table 1. Characteristics of the PtBuA brushes synthesized depending on the reaction time and the ratio of endfunctionalized chains to non-reactive chains (of the same molecular weight).

\begin{tabular}{|ccccc|}
\hline$M_{\mathrm{n}}\left(\mathrm{g} \mathrm{mol}^{-1}\right)$ & $\begin{array}{c}\text { Ratio of end-functionalized } \\
\text { chains to non-reactive chains } \\
(\%)\end{array}$ & $\begin{array}{c}\text { Reaction time } \\
(\mathrm{h})\end{array}$ & $\begin{array}{c}\text { Dry thickness, } \gamma \\
(\AA)\end{array}$ & $\begin{array}{c}\text { Grafting density, } \sigma \\
\left(\mathrm{nm}^{-2}\right)\end{array}$ \\
\hline 42000 & 10 & 1 & 40 & 0.060 \\
42000 & 100 & 1 & 83 & 0.125 \\
42000 & 100 & 24 & 93 & 0.140 \\
6500 & 100 & 1 & 43 & 0.418 \\
\hline
\end{tabular}

Some precautions had to be taken to prevent the thermal degradation of the PtBuA chains. The TGA curve for poly(tert-butyl acrylate) degradation is displayed in Figure 1. It shows that the weight loss, which corresponds to the conversion of the PtBuA into PAA chains, becomes significant for temperatures above $150{ }^{\circ} \mathrm{C}$ and is clearly confirmed at $200{ }^{\circ} \mathrm{C}$. The attachment step of the PtBuA chains on the surface by "grafting-onto" required heating above the glass transition temperature of the PtBuA $\left(T_{\mathrm{g}}=43^{\circ} \mathrm{C}\right)$ and, the reaction had to occur at high temperatures in order to obtain high rates for the esterification reaction between the epoxy-functionalized surface and the $\mathrm{COOH}$ terminated chains. As the degradation of PtBuA occurred at $150{ }^{\circ} \mathrm{C}$ and above, we settled for a thermal anchoring at $120^{\circ} \mathrm{C}$.

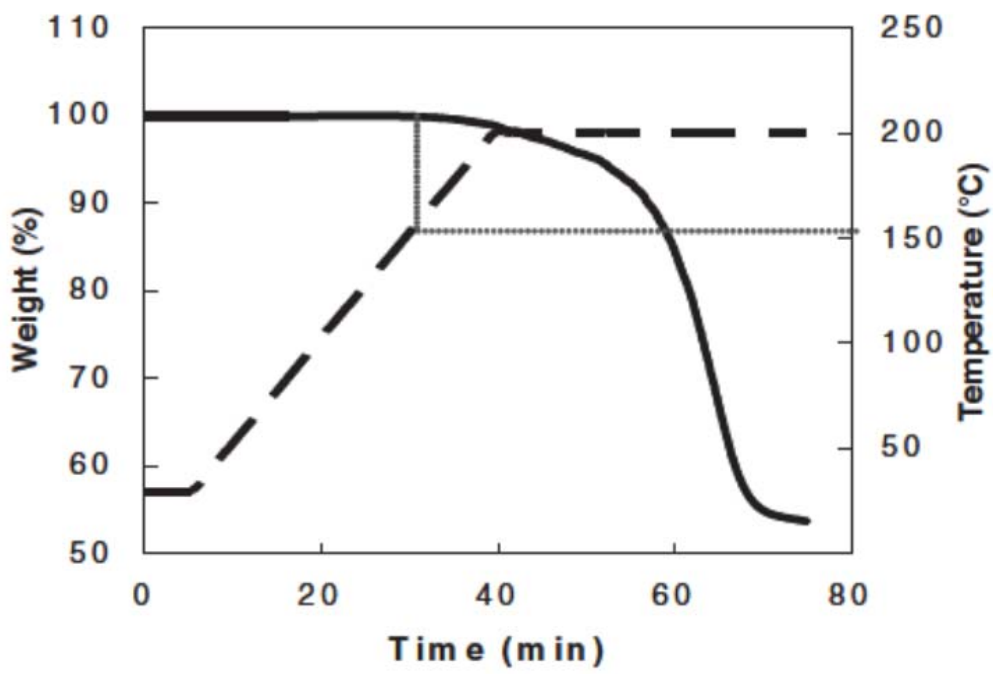

Figure 1. TGA curve of the PtBuA degradation. Both the weight loss (solid line) and the heating temperature (dotted line) are represented as functions of an arbitrary heating time.

This process avoids not only the conversion of tert-butyl acrylate into acrylic acid units but also the random anchoring of acrylic acid units on the GPS self-assembled monolayer during the annealing step. It resulted then in the sole grafting of PtBuA chains on the surface by the $\mathrm{COOH}$-functionalized extremity and the formation of extended brushes. In addition, the formation of loops was prevented by reacting $\mathrm{COOH}$-functionalized tert-butyl acrylate oligomers $\left(M_{\mathrm{n}}=4200 \mathrm{~g} \mathrm{~mol}^{-1}\right)$ with the GPS silane 
molecules that were not linked to long PtBuA chains. The thickness of the added short chains layers was about $4 \AA$. These short chains inhibited the reaction of the GPS molecules with random units of the long PAA chains during the pyrolysis process. This protection layer allowed the formation of extended brushes and avoided the formation of loops, as shown next. Indeed, in the bimodal brushes, the (very) short chains acted as a carpet and the structure of the brush was provided by the long chains. In addition, the oligomers operate as a protection layer for the anchoring points of the long chains.[11]

\subsection{The Hydrolysis Reaction for the Conversion into Poly(acrylic acid) Brushes}

Since poly(acrylic acid) brushes cannot be synthesized in organic media in a single step by the "grafting-onto" method, the strategy was first to synthesize poly(tert-butyl acrylate) brushes and then to remove the tert-butyl protecting group by an acid hydrolysis or a pyrolysis reaction in order to obtain the desired acrylic acid functionalities.

In a previous paper, we showed that the acid hydrolysis reaction could be aggressive for polymer brushes.[22] Two hydrolysis reagents were compared: hydrochloric acid and trifluoroacetic acid at $1.3 \mathrm{~mol} \mathrm{~L}^{-1}$ in aqueous solutions. The hydrolysis rate and the resistance rate were determined as a function of reaction time. We observed that total hydrolysis was always reached with an adverse degrafting of chains. The cleavage of the polymer chains induced by the acid hydrolysis is probably localized at the siloxane anchoring site, and not at the ester group (formed by the esterification reaction between the epoxy of the self-assembled monolayer and the carboxyl end-functionalization of the PtBuA chains). Actually, we demonstrated that the hydrolysis reaction does not damage the primary ester of the 2-(dimethylamino)ethyl methacrylate (DMAEMA) units in polyDMAEMA (PDMAEMA) brushes, but that the chains are degrafted. Lego et al. [24] synthesized PAA brushes covalently linked to mica by first growing PtBuA chains from the surface by ATRP and then hydrolyzing the PtBuA brushes. The hydrolysis was performed with a mixture of trifluoroacetic acid (10 vol\%) and dichloromethane (DCM). It was shown that the grafted layers are resistant to acid hydrolysis if trifluoroacetic acid is used. Boyes et al. [17] hydrolyzed tert-butyl acrylate to acrylic acid by refluxing the brush in a solution of $10 \%$ aqueous $\mathrm{HCl}$ for $1 \mathrm{~h}$ and $12 \mathrm{~h}$. Their samples were diblockcopolymer brushes, in which the inner block (close to the silica surface) was polystyrene or poly(methyl acrylate) and the outer block was tert-butyl acrylate. As expected, no degrafting of chains was observed since the hydrophobic block prevented the acid reagent from penetrating towards the surface and damaging the siloxane anchoring sites. Bergbreiter et al. [25] prepared poly(acrylic acid) grafts on polyethylene films by using $0.33 \mathrm{M}$ methanesulfonic acid in DCM for 25 min. This hydrolysis process allowed them to keep the same density of grafts, since the amide anchoring points were not sensitive to acid reagents. However, if homopolymers and silica surfaces are concerned, it seems that acid hydrolysis is not the most relevant approach (whatever the acid reagent used) to convert poly(tert-butyl acrylate) brushes into poly(acrylic acid) brushes without damaging them.

\subsection{The Pyrolysis Reaction for the Conversion into Poly(acrylic acid) Brushes}

The chemical deprotection of the tert-butyl ester group that can prevent the cleavage of chains is the pyrolysis approach.[18-20] The pyrolysis reaction was very simple since it consisted of heating the 
polymer brushes at $200^{\circ} \mathrm{C}$ under vacuum for $2 \mathrm{~h}$. As shown in Figure 1, the conversion of PtBuA into PAA chains clearly occurred at $200^{\circ} \mathrm{C}$.

Figure 2 shows the FTIR-ATR spectra of the PtBuA and PAA brushes before and after the pyrolysis reaction. We have also compared the spectra of the same PAA brush just after pyrolysis, and then after being dried after immersion in aqueous solution at $\mathrm{pH}=2$ and $\mathrm{pH}=9$.

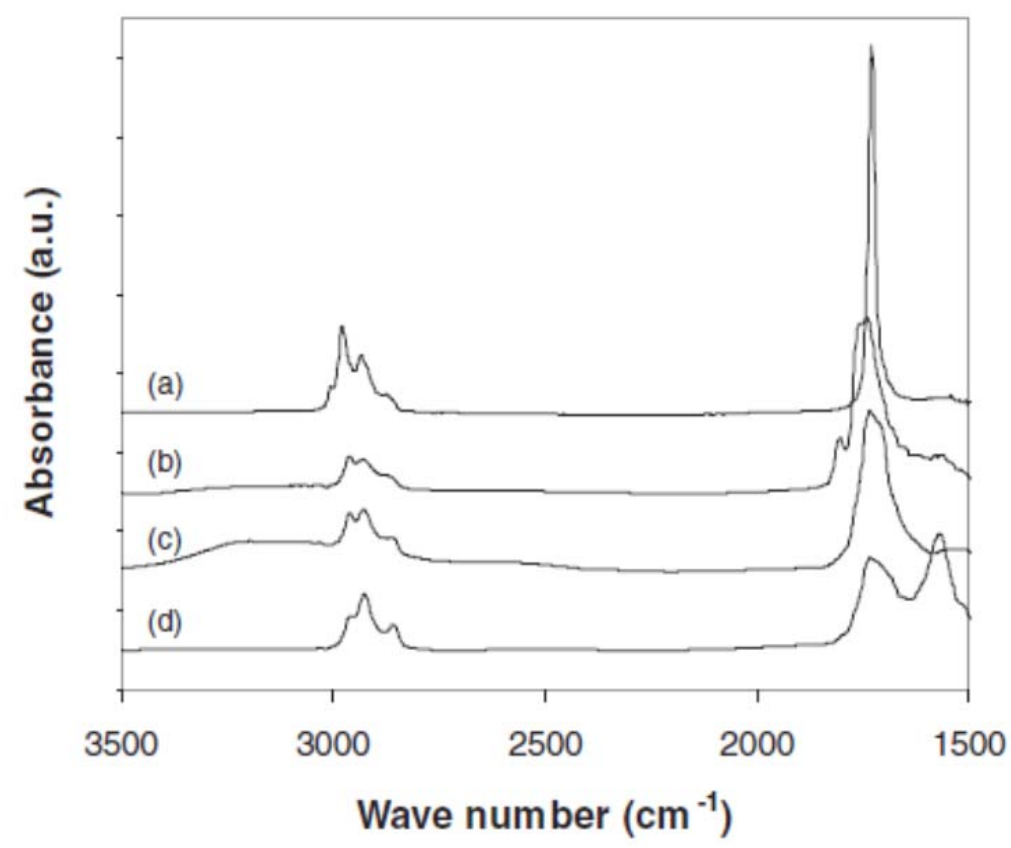

Figure 2. FTIR-ATR spectra of the PtBuA brush (a) and the PAA brush obtained after pyrolysis (b). Spectra are also shown for the PAA brush after it was put in aqueous solution at $\mathrm{pH}=2$ (c) and $\mathrm{pH}=9$ (d).

The deprotection of the tert-butyl ester group is indicated by the loss of the characteristic peak at $2977 \mathrm{~cm}^{-1}$ (asymmetric stretch of C-H of the tert-butyl acrylate) and the broadening of the peak at $1710 \mathrm{~cm}^{-1}$ ( $\mathrm{C}=\mathrm{O}$ stretch). The absorption bands at $1805 \mathrm{~cm}^{-1}$ and $1760 \mathrm{~cm}^{-1}$ are characteristic of the asymmetric and symmetric vibrations of the two $C=O$ groups of anhydrides. The presence of anhydrides was due to the dehydration of the PAA brushes (the ATR spectrum was recorded just after the removal of the samples from the oven at $200^{\circ} \mathrm{C}$ ). In fact, the anhydride functions could be softly hydrolyzed by immersion in a solution at $\mathrm{pH}=2$, as shown by the FTIR-ATR spectra. The loss of the absorption peaks of the anhydrides and the obvious presence of the broad bands between 3200 and $3700 \mathrm{~cm}^{-1}$ (corresponding to hydrogen-bonded $\mathrm{OH}$ groups) prove that this infrared spectrum is characteristic of a poly(acrylic acid) brush spectrum. The ionized polymer brush, dried after being immersed in a solution at $\mathrm{pH}=9$, shows the loss of the broad band characteristic of the $\mathrm{OH}$ group and the appearance of a peak at $1570 \mathrm{~cm}^{-1}$ that is associated with asymmetric stretching of the $\mathrm{CO}_{2}^{-}$ group. It should be pointed out that the absorbance of the bands at $2931 \mathrm{~cm}^{-1}$ and $2862 \mathrm{~cm}^{-1}$, associated with $\mathrm{CH}_{2}$ groups, remained the same for all of the spectra shown, indicating that no cleavage of chains occurred during the pyrolysis process.

The structure of the PtBuA and PAA brushes was also investigated by neutron reflectivity. This technique allows the determination of the brush density profile in a solvent (in the direction 
perpendicular to the surface). Figure 3 shows the neutron reflectivity curves of the PtBuA brush in $\mathrm{CDCl}_{3}$ and the PAA brush (obtained by pyrolysis for $2 \mathrm{~h}$ ) in $\mathrm{D}_{2} \mathrm{O}$ at $\mathrm{pH}=9$, and also the corresponding volume fraction profiles that best fit the reflectivity data. Note that the critical wave vector was shifted, since it depends on the solvent used. For the reflectivity at the silicon- $\mathrm{CDCl}_{3}$ interface, it was equal to $3.69 \times 10^{-3} \AA^{-1}$, whereas for the silicon- $D_{2} \mathrm{O}$ interface, the value was $7.38 \times 10^{-3} \AA^{-1}$. The PtBuA brush in chloroform was stretched up to $250 \AA$ from the surface and had a density profile that could be fitted with a parabolic function. This shape matches the profile of a polymer brush in a good solvent.[21] It should be noted that the profile of the bimodal (oligomers and long chains) PtBuA brush is similar to the profile of the monomodal brush with a very weak disturbance of the oligomer layer (data not shown). The PAA brush in aqueous solution at $\mathrm{pH}=9$ was also extended quite far from the surface. However, surprisingly the PAA chains appear to be less stretched than the PtBuA chains. Since the PAA chains in water at $\mathrm{pH}=9$ were ionized, the PAA brush was expected to behave as a polyelectrolyte brush and to be extended further from the surface than a polymer brush in a good solvent, such as the PtBuA brush. Indeed, the dry thickness obtained from the integral of the volume fraction profile of the PAA brush $(\gamma=38 \AA$ ) was predictably smaller than that of the PtBuA brush $(v=93 \AA)$ for the same grafting density, due to the mass loss of the tert-butyl groups, among others. In that case, it is more suitable to compare the volume fraction profiles of the brushes in a normalized representation by dividing the distance from the surface, $z$, by the dry thickness, $\gamma$. In this representation, the PAA brush was obviously more extended than the PtBuA brush (see inset in bottom graph in Figure 3). The stretching of the PAA and PtBuA chains could also be compared by calculating their degrees of swelling, which correspond to the ratio of the thickness of the brush in the presence of solvent to that of the dry brush without solvent. The degree of swelling for the PAA brush (equal to 4) was much higher than that for the PtBuA brush (equal to 1.8). It was also comparable to values found for polyelectrolyte brushes.[21] Accordingly, the density profiles obtained seem to confirm that the pyrolysis strategy was successful in converting the PtBuA brush into a PAA brush. This simple and rapid method has the advantage of avoiding the cleavage of chains from the surface. In summary, we have carefully demonstrated that the PAA layer obtained was an extended brush and not a layer with loops, by using neutron reflectivity. Actually, an adsorbed layer (with loops) should display a more abrupt volume fraction profile with a higher density near the surface, as shown next. Moreover, its degree of swelling cannot reach the value of 4 found here. [26]

We also emphasize that an appropriate duration of the pyrolysis is mandatory to obtain PAA brushes. We performed neutron reflectivity on PAA brushes of long chains (without short chains) obtained after a $24 \mathrm{~h}$ pyrolysis. Starting from $93 \AA$ A-thick PtBuA brushes, the PAA layer presented a thickness of $59 \AA$ for a $24 \mathrm{~h}$ pyrolysis (instead of $38 \AA$ for a $2 \mathrm{~h}$ pyrolysis). The neutron reflectivity curve of the PAA layer immersed in water equilibrated at $\mathrm{pH}=9$ is given in Figure 4, with the volume fraction of monomers corresponding to the best fit of the reflectivity curve. The results for PAA bimodal brushes are also presented after a $2 \mathrm{~h}$ pyrolysis, for comparison. The density profile of the sample that had undergone a $2 \mathrm{~h}$ pyrolysis is very soft, and very stretched away from the substrate. At the surface, the volume fraction of monomer was equal to 0.30 , which means that the polymer was totally swollen, with a swelling ratio equal to 4 . Comparatively, the sample obtained after a $24 \mathrm{~h}$ pyrolysis shows an unswollen layer with a very high monomer concentration leading to a swelling ratio equal to 2 . On top of the first layer, a second layer looks like the $2 \mathrm{~h}$ pyrolyzed sample. The density profile of the sample pyrolyzed for $24 \mathrm{~h}$ is incompatible with the formation of PAA brushes. 

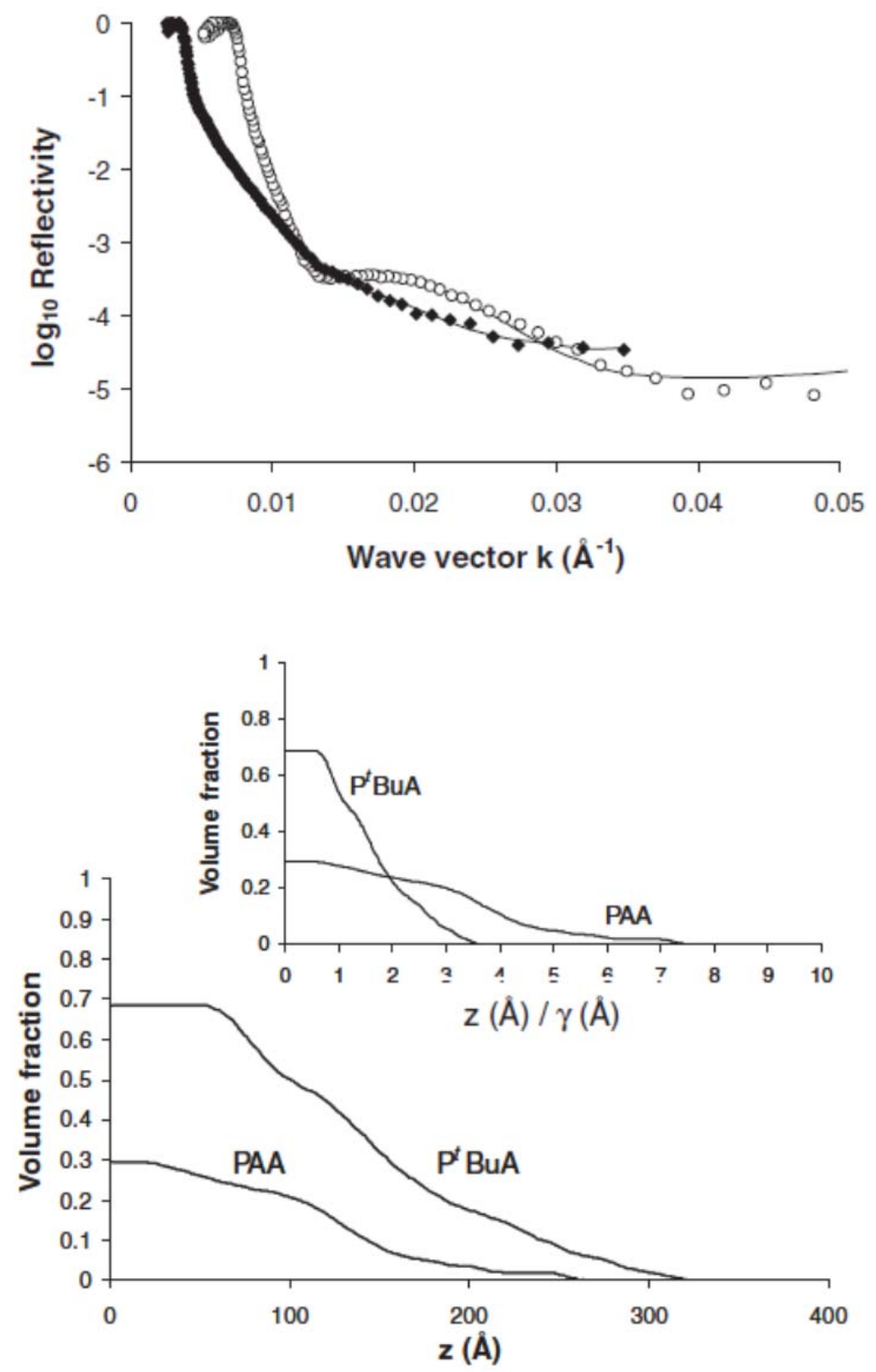

Figure 3. Top: neutron reflectivity data of the PtBuA brush in $\mathrm{CDCl}_{3}(\diamond)$ and of the PAA brush in $\mathrm{D}_{2} \mathrm{O}$ at $\mathrm{pH}=9$ (o). Bottom: corresponding monomer volume fraction profiles of the PtBuA and PAA brushes that best fit the reflectivity curves. Inset: volume fraction of the PtBuA and PAA brushes as a function of the normalized distance from the surface $(z / \gamma)$.

Actually, if the pyrolysis was not total for the $24 \mathrm{~h}$ pyrolysis and some PtBuA were to remain at the surface, then the volume fraction of monomers at the surface would be higher than 1: as PtBuA has a lower neutron scattering density than PAA and as the unit is assumed to be acrylic acid for the calculation of the density profile, a collapsed PtBuA layer would lead to a volume fraction of monomer equal to 1.45 . 

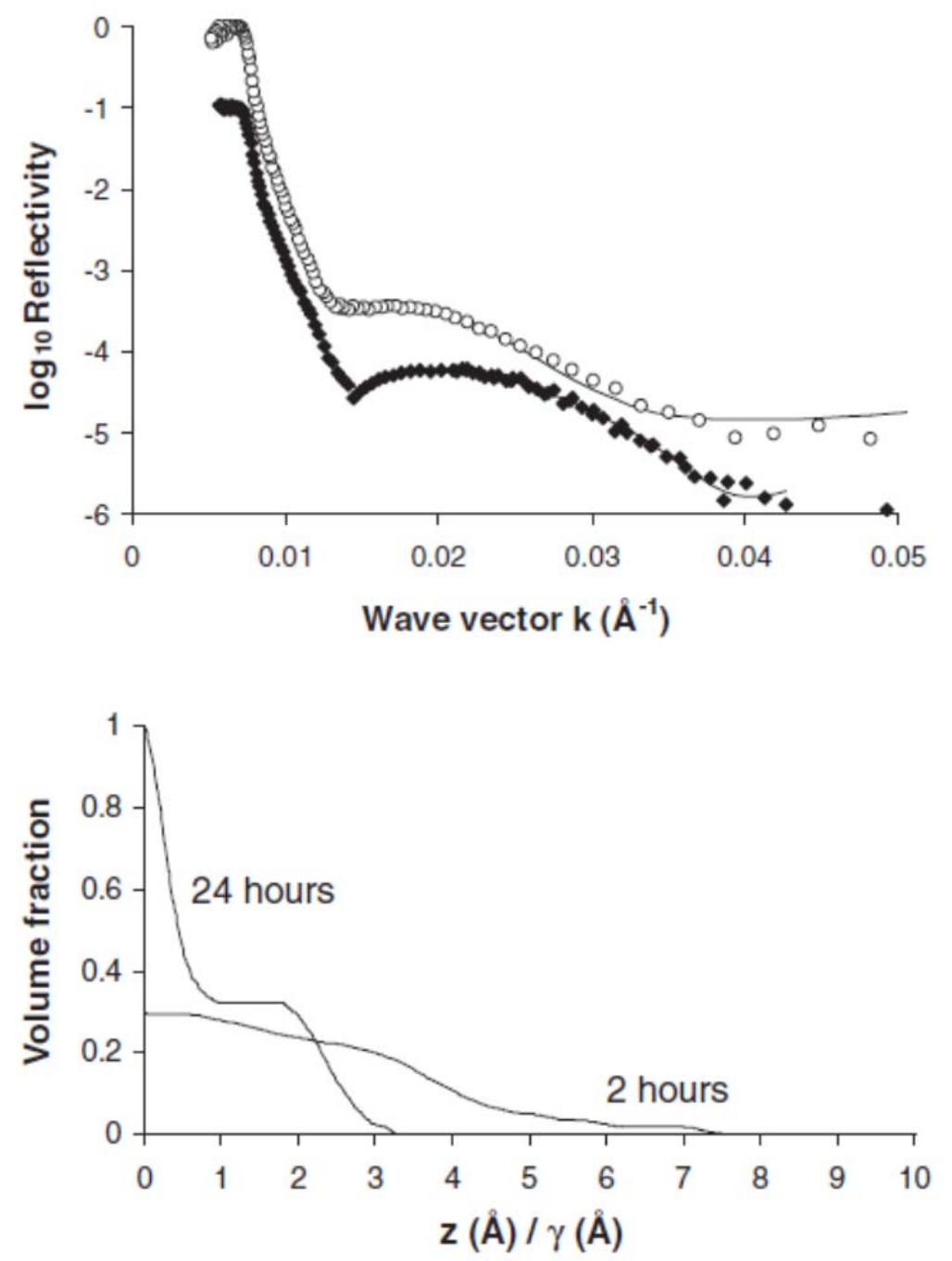

Figure 4. Top: neutron reflectivity data of the PAA brush obtained after $24 \mathrm{~h}$ pyrolysis $(\star)$ and the PAA bimodal brush after $2 \mathrm{~h}$ pyrolysis (O). Bottom: The corresponding monomer volume fraction profiles of the PAA brushes that best fit the reflectivity curves. The density profile is presented as a function of the normalized distance from the surface $(z / v)$. Both of the PAA brushes were in $\mathrm{D}_{2} \mathrm{O}$ at $\mathrm{pH}=9$.

We propose two side reactions to explain the dense zone near the surface. First, the acrylic acid units that are formed along the chain and able to react with unreacted epoxide still present at the surface may lead to the formation of loops instead of a brush. Second, the dehydration of two carboxylic acid functions can be the cause of the formation of anhydrides and then, a cross-linked material. To avoid the multiple covalent bonding of the PtBuA chains on the surface during pyrolysis at $200{ }^{\circ} \mathrm{C}$, one should carpet the epoxy-functionalized surface by the addition of small chains ( $\mathrm{Mn} \approx 4200 \mathrm{~g} \mathrm{~mol}^{-1}$ ) to hamper access to the surface by the long chains. In addition, a $2 \mathrm{~h}$ pyrolysis reaction is sufficient for the conversion of PtBuA brushes to PAA brushes.

\section{Conclusion}

We have reported the "grafting-onto" route to synthesize poly(acrylic acid) brushes covalently attached to planar silica surfaces. Each step of the synthesis was carefully characterized using 
complementary surface techniques such as ellipsometry, infrared spectroscopy and neutron reflectivity. The neutron reflectivity is, regrettably, not a usual characterization tool for the synthesis of polymer brushes, yet it is a powerful method for the structural characterization of polymer brushes. Here, it was perfectly appropriate, since it allowed an additional and valuable description of the brush. The "grafting-onto" method, which consists in the attachment of preformed chains, is a straightforward and cheap approach since it requires only commercial polymers like carboxyl endfunctionalized poly(tert-butyl acrylate) in small amounts. We have also shown that the pyrolysis reaction is a rapid and simple approach that allows the deprotection of the ester group and the conversion into polyacid brushes without the cleavage of chains. Nevertheless, some thermal precautions were cautiously taken to prevent the formation of loops, in order to obtain extended brushes. While these polyacid brushes are attractive for their potential applications in biological areas, they are also expected to have interesting properties regarding the design of surfaces with switchable adsorption and/or adhesion. Such work will be reported elsewhere.

\section{Acknowledgements}

The authors gratefully thank the French National Research Agency (ANR) for its financial support.

\section{References}

[1] Polymer Brushes: Synthesis, Characterization, Applications, (Eds: R. C. Advincula, W. J. Brittain, K. C. Caster, J. Rühe) Wiley-VCH, Weinheim, Germany 2004.

[2] J. Rühe, M. Ballauff, M. Biesalski, P. Dziezok, F. Gröhn, D. Johannsmann, N. Houbenov, N. Hugenberg, R. Konradi, S. Minko, M. Motornov, R. Netz, M. Schmidt, C. Seidel, M. Stamm, T. Stephan, D. Usov, H. Zhang, Adv. Polym. Sci. 2004, 165, 79.

[3] W. J. Brittain, S. Minko, J. Polym. Sci., Part A: Polym. Chem. 2007, 45, 3505.

[4] W. J. Brittain, S. G. Boyes, A. M. Granville, M. Baum, B. K. Mirous, B. Akgun, B. Zhao, C. Blickle, M. D. Foster, Adv. Polym. Sci. 2006, 198, 125.

[5] S. Minko, Polym. Rev. 2006, 46, 397.

[6] I. Luzinov, S. Minko, V. V. Tsukruk, Prog. Polym. Sci. 2004, 29, 635.

[7] S. P. Cullen, X. Liu, I. C. Mandel, F. J. Himpsel, P. Gopalan, Langmuir 2008, 24, 913.

[8] J. Dai, Z. Bao, L. Sun, S. U. Hong, G. L. Baker, M. L. Bruenong, Langmuir 2006, 22, 4274.

[9] N. Singh, X. Cui, T. Boland, S. M. Husson, Biomaterials 2006, 28, 763.

[10] Y. Tran, P. Auroy, L. T. Lee, Macromolecules 1999, 32, 8952.

[11] Y. Tran, P. Auroy, J. Am. Chem. Soc. 2001, 123, 3644.

[12] A. Sidorenko, S. Minko, K. Meuder, H. Dushner, M. Stamm, Langmuir 1999, 15, 8349.

[13] N. Houbenov, S. Minko, M. Stamm, Macromolecules 2003, 36, 5897.

[14] H. Karsten, D. Aulich, L. lonov, N. Esser, K. J. Eichhorn, M. Motornov, M. Stamm, S. Minko, Langmuir 2009, 25, 10987.

[15] T. Wu, P. Gong, I. Szleifer, P. Vlcek, V. Subr, J. Genzer, Macromolecules 2007, 40, 8756.

[16] Z. Bao, M. L. Bruening, G. L. Baker, J. Am. Chem. Soc. 2006, 128, 9056.

[17] S. G. Boyes, B. Akgun, W. J. Brittain, M. D. Foster, Macromolecules 2003, 36, 9539. 
[18] N. D. Treat, N. Ayres, S. G. Boyes, W. J. Brittain, Macromolecules 2006, 39, 26.

[19] N. Ayres, C. D. Cyrus, W. J. Brittain, Langmuir 2007, 23, 3744.

[20] N. Ayres, S. G. Boyes, W. J. Brittain, Langmuir 2007, 23, 182.

[21] S. Sanjuan, P. Perrin, N. Pantoustier, Y. Tran, Langmuir 2007, 23, 5769.

[22] S. Sanjuan, Y. Tran, J. Polym. Sci., Part A: Polym. Chem. 2008, 46, 4305.

[23] S. Sanjuan, Y. Tran, Macromolecules 2008, 41, 8721.

[24] B. Lego, W. G. Skene, S. Giasson, Macromolecules 2010, 43, 4384.

[25] D. E. Bergbreiter, J. G. Franchina, K. Kabza, Macromolecules 1999, 32, 4993.

[26] Polymers at Interfaces, (Eds: G. J. Fleer, M. A. Cohen Stuart, J. M. H. M. Scheutjens, T. Cosgrove,

B. Vincent), Chapman and Hall, Bristol, UK 1993. 